\title{
VOLUME, CHEEGER AND GROMOV
}

\author{
ITAI BENJAMINI
}

\begin{abstract}
It is shown that a manifold of bounded local geometry with Cheeger constant bigger than $h$ and Gromov hyperbolicity constant smaller than $\delta$ has either infinite volume or it's volume is bounded by a function depending only on $h$ and $\delta$ and the bounded geometry parameters.
\end{abstract}

\section{Introduction}

In this note we will restrict the discussion to the set of complete Riemannian manifolds $M$ of some fixed dimension $n$, with all sectional curvatures bounded from below by say -1 , and injectivity radius bigger than $r_{0}>0$. Our goal is to prove the following.

Theorem 1. Given $h, \delta>0$, assume the Cheeger constant of $M$ is bigger than $h$ and the Gromov hyperbolicity constant of $M$ is smaller than $\delta$, then either $M$ has infinite volume or it's diameter is bounded by $f(h, \delta)<\infty$, a function which depends only on $\delta$ and $h$ and the bounded geometry parameters.

Since we assume bounded geometry with fixed bounds, a bound on the diameter implys a bound on the volume.

The theorem was inspired by a related result from Benjamini (1998).

We start with definitions.

\section{Definition (Cheeger constant).}

$$
h(M)=\inf \frac{\operatorname{Area}(\partial A)}{\min \left(\operatorname{Vol}(A), \operatorname{Vol}\left(A^{c}\right)\right)},
$$

where $A$ runs over open subsets of $M$ with finite volume. $A^{c}$ is the compliment of $A, \partial A$ is the boundary of $A$, Area $(\partial A)$ denotes the $(n-1)$-dimensional volume of $\partial A$, and Vol denotes $n$-dimensional volume.

Definition ( $\delta$-hyperbolic). Let $M$ be a manifold. Given three points $a, b, c \in$ $M$, pick geodesics between any two to get a geodesic triangle. Denote the geodesics by $[a, b],[a, c],[b, c]$. Say the triangle is $\delta$-thin if for any $p \in[a, b]$

$$
\min (d(p,[a, c]), d(p,[b, c])) \leq \delta,
$$

and the same for $p \in[a, c]$ or $[b, c] . M$ is said to be $\delta$-hyperbolic if all geodesic triangles in $M$ are $\delta$-thin. Let

$$
\delta(M)=\inf \{\delta \mid M \text { is } \delta \text {-thin }\} .
$$

Received May 4, 1998. 
Note that the real hyperbolic spaces $\mathbb{H}^{n}$ have infinite volume, strictly positive Cheeger constant and finite hyperbolicity constant.

\section{Proof}

Proof. Given $M$ let $h=h(M), \delta=\delta(M)$ and assume that $d$ is chosen so that for any ball $B(a, r) \subset M$ with radius $r>1, \operatorname{Vol}(B(a, r))<d^{r}$. Such a $d$ exists and depends only on the dimension and the bounded geometry conditions we assumed at the start. See for instance Chavel (1993).

From now on we will assume $M$ has finite volume. Since $M$ has bounded geometry it is compact. Set $R=\log _{d} \operatorname{Vol}(M)$ and pick $C>0$ depending only on $h$ and $d$ such that

$$
(h / 2) \operatorname{Vol} B(a,(1 / 2-2 C) R)>d^{2 C R},
$$

for any ball centered at any $a \in M$.

Let $a, b$ be two points that realize the diameter of $M$, and $\gamma$ a geodesic between $a$ and $b$. Let $m$ be the midpoint of $\gamma$. Pick a ball $B$ of radius $r^{\prime} C R \leq r^{\prime} \leq 2 C R$ around $m$, for which $\operatorname{Area}(\partial B)<d^{2 C R}$. Such an $r^{\prime}$ exists because of the volume upper bound in terms of $d$ and the fact that $\operatorname{Vol}(B)=\int_{0}^{r^{\prime}} \operatorname{Area}(\partial B(m, r)) d r$. The distance from $a$ to $b$ is at least $R$. Hence the distance from $a$ to $B$ is at least $(1 / 2-2 C) R$. The same is true for the distance from $b$ to $B$. Let $M \backslash B$ be the manifold with boundary obtained from $M$ by cutting $B$ off. Denote by $d_{M \backslash B}$ the distance function on $M \backslash B$. By Gromov (1987) 7.1.A there is $c_{\delta}>1$ such that

$$
d_{M \backslash B}(a, b) \geq c_{\delta}^{C R},
$$

(the actual bound in Gromov (1987) is $\left.\delta\left(2^{C R / \delta}-2\right)\right)$. Now assume

$$
\operatorname{Vol}_{M \backslash B}\left(B\left(a, d_{G \backslash B}(a, b) / 2\right)\right) \leq \operatorname{Vol}_{M \backslash B}\left(B\left(b, d_{G \backslash B}(a, b) / 2\right)\right) .
$$

That is, the Volume of the ball in $M \backslash B$ centred at $a$ of half the distance in $M \backslash B$, from $a$ to $b$, is not bigger than the volume of the similar ball centered at $b$. Now let $A(n)=B_{M \backslash B}(a, n) \backslash B_{M \backslash B}(a, n-1)$. Thus, for any $n<d_{M \backslash B}(a, b) / 2$, by integrating the areas of $\partial\left(B_{M \backslash B}(a, r)\right), n-1 \leq r \leq n$,

$$
\operatorname{Vol}(A(n)) \geq h \operatorname{Vol}\left(B_{M \backslash B}(a, n-1)\right)-\operatorname{Area}(\partial B) .
$$

Yet $C$ was chosen so that for $r \geq(1 / 2-2 C) R$,

$$
\operatorname{Area}(\partial B) \leq(h / 2) \operatorname{Vol} B(a,(1 / 2-2 C) R) \leq(h / 2) \operatorname{Vol}\left(B_{G \backslash B}(a, r)\right) .
$$

(For $r<(1 / 2-2 C) R), B_{M \backslash B}(a, r)$ is disjoint from $B$ ). So for $n \leq d_{M \backslash B}(a, b) / 2$,

$$
\operatorname{Vol}\left(B_{M \backslash B}(a, n)\right)>(1+h / 2) \operatorname{Vol}\left(B_{M \backslash B}(a, n-1)\right) .
$$


We get then,

$$
\begin{aligned}
d^{R} \geq \operatorname{Vol}(M) & \geq(1+h / 2)^{d_{M \backslash B}(a, b) / 2} \\
& \geq(1+h / 2)^{c_{\delta}^{C R} / 2},
\end{aligned}
$$

which forces an upper bound on the diameter $R$ in terms of $d, h$ and $\delta$.

\section{Acknowledgement}

As always, thanks to Oded Schramm for useful advice.

\section{References}

[1] I. Benjamini, Expanders are not hyperbolic, Israel J. Math., to appear.

[2] I. Chavel, Riemannian geometry, a modern introduction, Cambridge Tracts in Mathematics, 108, Cambridge University Press, Cambridge, 1993.

[3] M. Gromov, Hyperbolic groups, Essays in group theory, pp. 75-263, Math. Sci. Res. Inst. Publ. 8, Springer, 1987.

Weizmann Institute, Rehovot, IsRael 76100

E-mail address: itai@wisdom.weizmann.ac.il 